\title{
Glasdegib in combination with low-dose Cytarabine for the outpatient treatment of relapsed or refractory acute myeloid leukemia in unfit patients
}

\author{
Andrius Zucenka $^{1,2} \cdot$ Kazimieras Maneikis ${ }^{2} \cdot$ Birute Pugaciute $^{2} \cdot$ Ugne Ringeleviciute $^{2}$ - Austeja Dapkeviciute ${ }^{2}$. \\ Linas Davainis $^{2}$ • Guoda Daukelaite ${ }^{1}$ • Paulina Burzdikaite ${ }^{1}$ - Vytautas Staras ${ }^{2}$ - Laimonas Griskevicius ${ }^{1,2}$
}

Received: 29 January 2021 / Accepted: 18 February 2021 / Published online: 4 March 2021

(C) The Author(s), under exclusive licence to Springer-Verlag GmbH, DE part of Springer Nature 2021

\begin{abstract}
We retrospectively collected clinical data on 31 relapsed or refractory acute myeloid leukemia (R/R AML) patients who were treated with outpatient glasdegib and low-dose Cytarabine (LDAraC) at our institution. The median age was 67 years (45-86). The median Eastern Cooperative Oncology Group performance status was $2(1-3)$. The patients had previously received a median number of $2(1-4)$ treatment lines, $61 \%$ (19/31) had been treated with intensive chemotherapy, 29\% (9/31) had relapsed after allogeneic stem cell transplantation, and 45\% (14/31) had had venetoclax exposure. Adverse cytogenetics were identified in $45 \%$ (14/31) of the cases. The CR + CRp rate was $21 \%$ (6/29) among evaluable patients. The median overall survival was 3.9 months for all patients. Different median overall survival times were observed in responders, patients achieving stable disease and those diagnosed with progressive disease: not reached vs 3.9 months vs 0.8 months, respectively $(p<0.001)$. The most common adverse events were pneumonia $(29 \%, 9 / 31)$, sepsis $(23 \%, 7 / 31)$, and febrile neutropenia $(16 \%, 5 / 31)$. Glasdegib + LDAraC is a fairly safe, non-intensive, outpatient regimen inducing complete remission and resulting in prolonged survival in some R/R AML patients.
\end{abstract}

Keywords AML $\cdot$ Glasdegib $\cdot$ Relapsed $\cdot$ Refractory $\cdot$ Venetoclax

\section{Introduction}

The prognosis of relapsed or refractory acute myeloid leukemia (R/R AML) in elderly and unfit population remains poor as intensive salvage chemotherapy regimens cannot be administered due to excessive toxicity whereas standard lowintensity therapies often lack efficacy $[1,2]$. During the last decade, a number of targeted therapies have shown promising efficacy in frontline and R/R AML settings including elderly and frail patients [3, 4]. Glasdegib is a novel small-molecule inhibitor of the Smoothened (SMO) receptor, and its efficacy is based on downregulation of the Hedgehog signaling

Andrius Zucenka

andrius.zucenka@santa.lt

1 Faculty of Medicine, Institute of Clinical Medicine, Vilnius University, Vilnius, Lithuania

2 Bone Marrow Transplantation Department, Hematology, Oncology and Transfusion Medicine Center, Vilnius University Hospital Santaros Klinikos, 08661 Vilnius, Lithuania pathway which is involved in the resistance of AML cells [5-7]. Glasdegib combined with low dose Cytarabine (LDAraC) is currently approved for newly diagnosed AML patients unfit for standard chemotherapy [8]. The pivotal phase II BRIGHT AML 1003 trial demonstrated a higher complete remission rate (17\% vs $2.3 \%)$ and significantly longer median overall survival ( 8.8 vs 4.9 months) in unfit newly diagnosed AML patients receiving Glasdegib + LDAraC compared to the comparator arm treated with LDAraC only [9]. Cortes et al. had also presented positive efficacy, safety, and survival outcomes of AML/high-risk myelodysplastic syndrome (HR-MDS) patients treated with glasdegib in combination with standard induction $7+3$ [10]. Glasdegib is currently being evaluated in a phase III trial in combination with either azacitidine or standard induction $7+3$ (BRIGHT AML 1019) for previously untreated AML and several other phase I-II studies [11]. Nevertheless, glasdegib data in the R/R AML setting are lacking. We present the results of 31 heavily pretreated, elderly, and unfit R/R AML patients who were treated with glasdegib + LDAraC. 


\section{Methods}

We retrospectively collected clinical data on R/R AML patients who were treated with glasdegib + LDAraC in 20182020 at Vilnius University Hospital Santaros Klinikos, Vilnius, Lithuania. Glasdegib was provided via a Compassionate Use Program by Pfizer, and the treatment was approved by the local Ethics Committee on a case by case basis. The data collection was approved by Vilnius Regional Biomedical Research Ethics Committee. All patients were at least 18 years of age, had R/R AML with no active infection or life-threatening organ toxicity, and had all treatment options exhausted.

\section{Glasdegib + LDAraC}

The treatment cycles consisted of self-administered Cytarabine $20 \mathrm{mg} / \mathrm{m}^{2} \mathrm{~s} / \mathrm{c}$ days $1-10$ and glasdegib $100 \mathrm{mg}$ $\mathrm{p} / \mathrm{o}$ daily on an outpatient basis. The cycles were repeated every 28-35 days; longer interruptions were allowed in case of toxicity or social reasons. Glasdegib was continued after day 28 even if the start of the next cycle was postponed. Indications for stopping the treatment were progressive disease with no further clinical benefit observed by the treating physician, and excessive toxicity or availability of alternative treatment strategies. Routine ECG evaluation every 1-4 weeks and potassium \pm magnesium supplements were administered to reduce the risk of QT prolongation in the majority of cases. Strong CYP3A4 inhibitors or drugs prolonging the QT interval were allowed if alternatives were not available; however, ECG evaluation was repeated more frequently in such cases.

\section{Endpoints and statistical analysis}

Descriptive statistics were used for baseline characteristics, safety, and efficacy analysis. For efficacy analysis, we calculated the overall response rate (ORR), which consisted of complete remission (CR), complete remission with incomplete hematological recovery (CRi), and complete remission with incomplete platelet recovery (CRp) or partial remission (PR) as defined by the European Leukemia Network 2017 (ELN 2017) criteria [12]. We calculated day 30 and day 60 mortality and recorded grade 3-5 nonhematological adverse events according to the Common Terminology Criteria of Adverse Events (CTCAE v5.0) scale. For additional toxicity evaluation, we also analyzed the prevalence of dysgeusia, anorexia, alopecia, QT prolongation, and gastrointestinal symptoms as adverse events of special interest related to glasdegib.

Fisher's exact and Mann-Whitney $U$ tests were used for comparison of ordinal and numerical data between the groups. Kaplan-Meier analysis was used for overall survival (OS). OS was estimated from treatment start to death from any cause with live patients censored on their last follow-up date. Reverse Kaplan-Meier analysis was used for median follow-up time evaluation. The log-rank test was used for the intergroup comparison of survival data. Univariate Cox regression analysis was used to evaluate the effect of different variables on OS. Data cutoff date was November 16, 2020. Difference with $p<0.05$ was considered statistically significant.

\section{Results}

Thirty-one patients (17 female) were included in the study. Detailed baseline characteristics are summarized in Table 1 . The median age was 67 years $(45-86) ; 48 \%(15 / 31)$ of the patients were older than 70 years. The median Eastern Cooperative Oncology Group performance status (ECOG PS) was 2 (1-3). The patients had previously received a median number of 2 treatment lines; 29\% (9/31) had been treated with 3 or 4 lines of therapy. Sixty-one percent (19/ 31) had previously received intensive chemotherapy regimens and $13 \%(4 / 31)$ had primary refractory AML. Twenty-nine percent (9/31) had relapsed after allogeneic stem cell transplantation (alloSCT). The median time from alloSCT to relapse was 89 days (51-3177) and all but one had relapsed within 12 months of alloSCT. Thirty-nine percent $(12 / 31)$ had received only low-intensity therapies (hypomethylating agents (HMA)/LDAraC \pm venetoclax). Of note, $45 \%$ (14/31) had previous venetoclax exposure in combination with various regimens. Adverse cytogenetics were identified in $45 \%$ (14/31) of patients, of whom 5 (16\%) had complex karyotype. The most common mutations were IDH1/2 (23\%, 7/31), NPM1 (16\%, 5/31), ASXL1 (13\%, 4/31), RUNX1 (13\%, 4/31), NRAS/KRAS $(10 \%, 3 / 31)$, FLT3-ITD/TKD (10\%, 3/31), and TP53 (10\%, $3 / 31)$. Based on the ELN2017 risk group stratification criteria, $16 \%(5 / 31), 26 \%(8 / 31)$, and $58 \%(18 / 31)$ of the patients were stratified into favorable, intermediate, and adverse risk groups, respectively.

\section{Glasdegib + LDAraC treatment}

A median of 2 (1-7) glasdegib + LDAraC cycles were administered. Twenty-nine percent (9/31) of the patients received at least 4 cycles. Additional hydroxyurea was used in $16 \%$ $(5 / 31)$ of cases due to hyperproliferation. Treatment was stopped due to disease progression $(55 \%, 17 / 31)$, toxicity $(6 \%, 2 / 31)$, death in aplasia $(6 \%, 2 / 31)$, and alloSCT $(3 \%$, $1 / 31)$. Nine patients $(29 \%)$ continue glasdegib + LDAraC treatment (Fig. 1). Ninety-three percent (7/104) of all glasdegib + LDAraC cycles were administered in the outpatient setting. 
Table 1 Baseline characteristics

$n / N(\%)$

Gender

Male

Female

Age

Median age (range)

Age $\geq 60$

Age $\geq 70$

ECOG status

ECOG, median (range)

ECOG 1

ECOG 2

ECOG 3

Diagnosis

De novo AML

Secondary AML

Post-MDS/MPN

Therapy-related AML

Karyotype

Non-adverse

Adverse

Complex

ELN2017 risk group

Favorable

Intermediate

Adverse

AML characteristics

Bone marrow blast count, median (min-max)*

Proliferative disease**

Extramedullar AML

Number of previous treatment lines

Median (range)

2

Previous treatment

Previous intensive chemotherapy

Primary refractory (after 1 or 2 induction cycles)

Previous low-intensity therapy only (HMA/LDAraC \pm venetoclax)

Previous venetoclax exposure

Previous alloSCT

Median time from alloSCT to relapse, days (range)

Relapse within 12 months of alloSCT

$14 / 31(45 \%)$

$17 / 31(55 \%)$

$67(45-86)$

$21 / 31(68 \%)$

$15 / 31(48 \%)$

2 (1-3)

$14 / 31(45 \%)$

$12 / 31(39 \%)$

$5 / 31(16 \%)$

15/31 (48\%)

$16 / 31(52 \%)$

4/31 (13\%)

$12 / 31(39 \%)$

$17 / 31(55 \%)$

$14 / 31(45 \%)$

$5 / 31(16 \%)$

$5 / 31(16 \%)$

$8 / 31(26 \%)$

$18 / 31(58 \%)$

27\% (1-94)

9/31 (29\%)

$8 / 31(26 \%)$

$2(1-4)$

$11 / 31(35 \%)$

$11 / 31(35 \%)$

$8 / 31(26 \%)$

$1 / 31(3 \%)$

$19 / 31(61 \%)$

$4 / 31(13 \%)$

$12 / 31(39 \%)$

14/31 (45\%)

9/31 (29\%)

$89(51-3177)$

$8 / 9$ (89\%)

*2 patients had extramedullar $\mathrm{R} / \mathrm{R}$ AML

**Proliferative disease criteria: WBC $>10 \times 109 / 1$ with blasts and rapidly progressing leukocytosis and/or rapidly progressing extramedullar lesions

ELN European Leukemia Network, ECOG Eastern Cooperative Oncology Group performance status, AlloSCT allogeneic stem cell transplantation, $A M L$ acute myeloid leukemia, $H M A$ hypomethylating agents, $L D A r a C$ low-dose Cytarabine, $M D S$ myelodysplastic syndrome, $M P N$ myeloproliferative neoplasm

\section{Response evaluation and survival}

Response was evaluated in 94\% (29/31) of cases. Two patients had died during cycle 1 before response assessment. Detailed response evaluation is summarized in Table 2 and
Fig. 1. CR and CRp were confirmed in $17 \%(5 / 29)$ and $3 \%$ $(1 / 29)$ of the patients, respectively, and one patient achieved a PR. The composite CR + CRp rate was $21 \%(6 / 29)$, and the overall response rate $(\mathrm{CR}+\mathrm{CRi}+\mathrm{CRp}+\mathrm{PR})$ was $24 \%$ $(7 / 29)$. The median response duration was 2 months $(0.3--$ 6). Stable disease was achieved in $48 \%(14 / 29)$ of patients whereas the remaining $28 \%(8 / 29)$ were diagnosed with progressive AML during treatment. Characteristics of responding and non-responding patients are shown in Table 3. Responders were older and less exposed to intensive chemotherapy compared to non-responders. Survival data are summarized in Figs. 2 and 3. The median overall survival was 3.9 months for all the patients. The median follow-up time was 9.4 months. Different median survival lengths were observed in responders $(\mathrm{CR} / \mathrm{CRp} / \mathrm{PR})$, patients achieving $\mathrm{SD}$, and progressive cases: not reached vs 3.9 months vs 0.8 months, respectively $(p<0.001)$. In a univariate Cox regression analysis (Fig. 4$)$, older age $(\geq 70$ years) and previous non-intensive treatment only were associated with longer survival. Previous alloSCT, hyperproliferation, ECOG status, more than 1 previous treatment line, adverse cytogenetics, or previous venetoclax exposure did not have a significant impact on survival.

\section{Toxicity}

Grade 3-5 non-hematological toxicity analysis and any grade adverse events of special interest (AESI) are summarized in Table 4. The following infectious complications were the most common: pneumonia $(29 \%, 9 / 31)$, sepsis $(23 \%, 7 / 31)$, and febrile neutropenia $(16 \%, 5 / 31)$. COVID-19 was diagnosed in $6 \%$ of cases $(2 / 31)$ with one fatal outcome. Of note, grade $3-5$ bleeding events were also relatively common: $16 \%$ (3/31) of the patients suffered from gastrointestinal bleeding, $10 \%(3 / 31)$ had epistaxis, and 1 patient died due to central nervous system hemorrhage. Adverse events of special interest related to glasdegib were QT prolongation $(13 \%, 4 / 31)$, diarrhea $(6 \%, 2 / 31)$, alopecia $(6 \%, 2 / 31)$, gastritis $(6 \%, 2 / 31)$, anorhexia $(6 \%, 2 / 31)$, and dysgeusia $(3 \%, 1 / 31)$. Day 30 and day 60 all-cause mortality rates were $23 \%$ (7/31) and $29 \%$ $(9 / 31)$, respectively.

\section{Discussion}

Efficacy and safety data of glasdegib + LDAraC in R/R AML setting are limited to only several reports. A case series published by Tavares et al. evaluated six elderly patients with R/R AML or HR-MDS who had previously received a median of 2 therapy lines [13]. Four patients achieved a stable disease whereas a progressive disease was diagnosed in the remaining 2 cases. No objective 
Swimmer plot

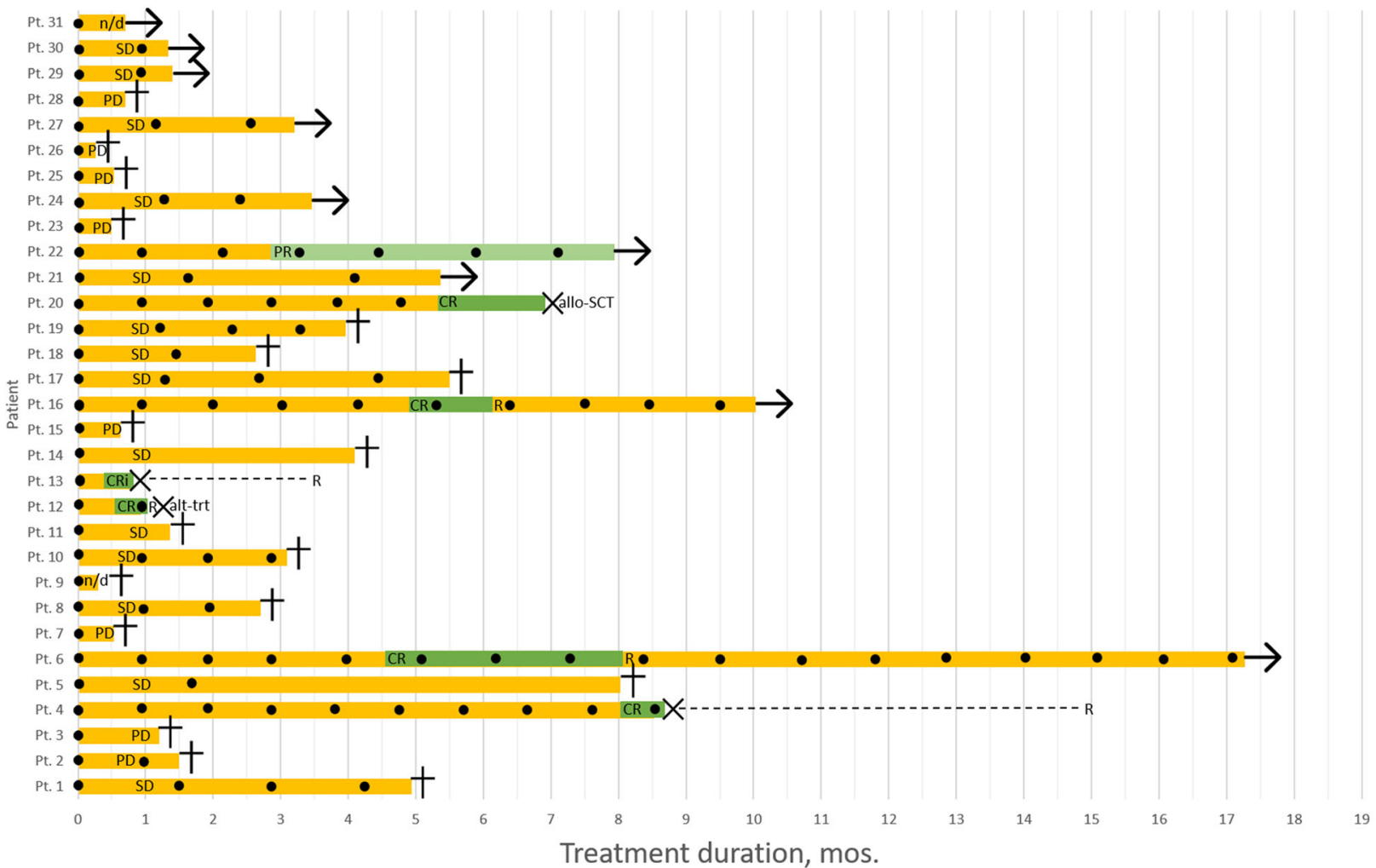

$\begin{array}{llll}\rightarrow \text {-treatment ongoing } & \bullet \text {-start of cycle } & \text { CR - complete remission } & \text { SD - stable disease } \\ & & \text { CRi-complete remission with incomplete recovery } & \text { PD - progressive disease } \\ \text { X-treatment terminated } & \text { 十-death } & \text { PR-partial remission } & \text { R-relapse }\end{array}$

$\mathrm{n} / \mathrm{d}$ - response not evaluated allo-SCT - allogeneic stem cell transplantation alt-trt - alternative treatment initiation

Fig. 1 Swimmer plot of $31 \mathrm{R} / \mathrm{R}$ AML patients treated with glasdegib + LDAraC

responses were observed; however, $4 / 6$ of the patients survived longer than 6 months. Salmman et al. evaluated the efficacy of glasdegib monotherapy in $\mathrm{R} / \mathrm{R}$ chronic myelomonocytic leukemia, myelodysplastic syndromes, or oligoblastic (20-30\% blasts in the bone marrow) AML in a phase II study [14]. A total of 35 (mostly MDS, 4 with AML) patients (median age 73 years) were included. Stable disease was achieved in $56 \%$ of the patients, and $6 \%$ had hematological improvement (HI). No objective responses (CR/CRi/CRp/PR/MLFS) were observed. The median OS was 10.4 months for the whole group and 20.6 months for patients achieving SD or HI.

Our patients treated with glasdegib + LDAraC were also elderly and unfit; however, all of them had R/R AML, approximately half had secondary AML and/or adverse cytogenetics. Importantly, the majority had previously failed multiple lines of intensive chemotherapy, with some relapsing very early after alloSCT. The remaining patients were refractory to HMA or LDAraCbased therapies. Venetoclax was used in almost half of the cases as well. Unsurprisingly, the median survival of our poor prognosis group was only 3.9 months. We demonstrate a $21 \%$ composite CR + CRp rate which compares favorably to both $\mathrm{R} / \mathrm{R}$ setting reports (no $\mathrm{CR} / \mathrm{CR} \mathrm{i} / \mathrm{CRp} /$ PRs) and the pivotal phase II study in previously untreated AML/HR-MDS (17\% CR rate) [9, 13, 14]. Achieving response $(\mathrm{CR} / \mathrm{CRp} / \mathrm{PR})$ translated into a prolonged $\mathrm{OS}$ (all 7 responders are alive after the median follow-up time of 9.4 months, 2 are in remission (1 was bridged to alloSCT and 1 is still on Gilteritinib), and 5 relapsed). Three out of five of the relapsed patients are still receiving glasdegib + LDAraC without overt AML progression signaling the importance of continuous treatment beyond relapse. Almost half of our patients achieved AML stabilization and survived longer when compared to cases with progressive disease. Though debatable, this may suggest a 
Table 2 Response evaluation

\begin{tabular}{ll}
\hline & $n / N(\%)$ \\
\hline Response evaluated & $29 / 31(94 \%)$ \\
Response not evaluated & $2 / 31(6 \%)$ \\
Best response & \\
CR & $5 / 29(17 \%)$ \\
CRp & $1 / 29(3 \%)$ \\
PR & $1 / 29(3 \%)$ \\
SD & $14 / 29(48 \%)$ \\
PD & $8 / 29(28 \%)$ \\
CR + CRp & $6 / 29(21 \%)$ \\
CR + CRp + PR & $7 / 29(24 \%)$ \\
Response (CR, CRp, PR) in subgroups & \\
Adverse cytogenetics & $2 / 13(15 \%)$ \\
Non-adverse cytogenetics & $5 / 16(31 \%)$ \\
Proliferative disease & $1 / 9(11 \%)$ \\
Post-alloSCT & $0 / 9(0 \%)$ \\
Previous intensive chemotherapy & $1 / 18(6 \%)$ \\
Primary refractory & $1 / 4(25 \%)$ \\
Previous low-intensity therapy only & $6 / 11(55 \%)$ \\
(HMA/LDAraC \pm venetoclax) & $2 / 14(28 \%)$ \\
Previous venetoclax exposure &
\end{tabular}

$C R$ complete remission, $C R p$ complete remission with incomplete platelet recovery, $P R$ partial remission, $P D$ progressive disease, $S D$ stable disease, alloSCT allogeneic stem cell transplantation, $H M A$ hypomethylating agents, LDAraC low-dose Cytarabine

benefit of glasdegib + LDAraC in patients without objective response as already noted in the frontline and $\mathrm{R} / \mathrm{R}$ glasdegib studies [13-15].

Interestingly, adverse karyotype was not associated with worse OS in our group, which differs from the data

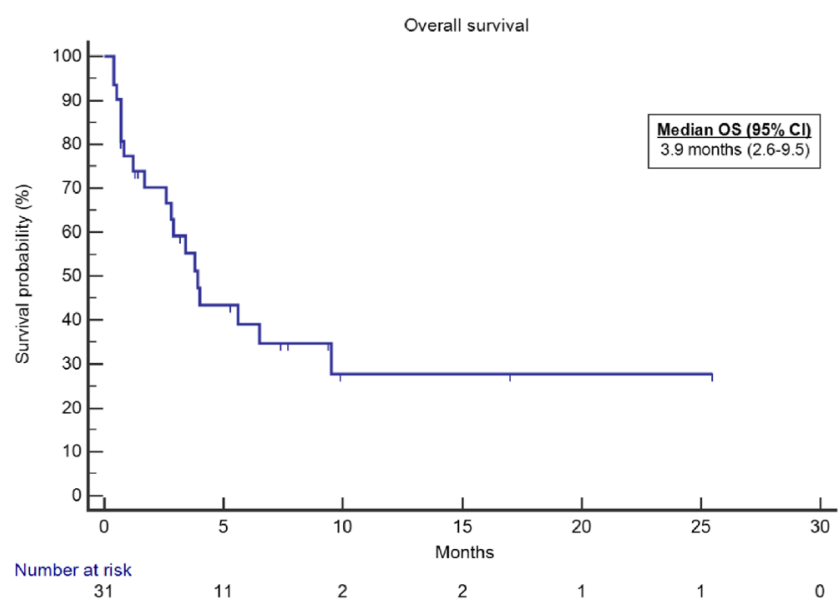

Fig. 2 Overal survival of $31 \mathrm{R} / \mathrm{R}$ AML patients treated with glasdegib + LDAraC. OS, overall survival; CI, confidence interval

of the BRIGHT AML 1003 study [9]. Of note, previous alloSCT, hyperproliferation, number of failed treatment lines, previous venetoclax exposure or, worse ECOG PS did not impact the OS in our analysis either. On the other hand, older age and previous exposure to only lowintensity therapies (HMAs/LDAraC \pm venetoclax) were associated with longer survival. Furthermore, the ORR in non-intensively pretreated patients was 55\% (6/11). This may indicate the possible benefit of glasdegib + LDAraC therapy in a subgroup of elderly R/R AML patients failing HMA treatment. These patients usually face poor prognosis due to the lack of effective salvage therapies if targetable FLT3 or IDH1/2 mutations are not present [16-18]. Our previously published data and the results of this study show that glasdegib + LDAraC may have a role in AML patients failing venetoclax [19].
Table 3 Characteristics of responders and non-responders

\begin{tabular}{lccc}
\hline & Responders (7) & Non-responders (22) & $p$ \\
\hline Age (median, range) & $75(67-86)$ & $64(45-84)$ & 0.020 \\
Number of previous treatment lines (median, range) & $1(1-3)$ & $2(1-4)$ & 0.146 \\
Adverse karyotype & $2 / 7(29 \%)$ & $11 / 22(50 \%)$ & 0.410 \\
ECOG (median, range) & $2(1-3)$ & $2(1-3)$ & 0.889 \\
Hyperproliferation & $1 / 7(14 \%)$ & $8 / 22(36 \%)$ & 0.381 \\
Previous intensive chemotherapy & $1 / 7(14 \%)$ & $17 / 22(77 \%)$ & 0.005 \\
Post-alloSCT & $0(0 \%)$ & $9 / 22(41 \%)$ & 0.066 \\
Previous venetoclax exposure & $2 / 7(29 \%)$ & $12 / 22(55 \%)$ & 0.389 \\
\hline
\end{tabular}

Proliferative disease criteria: WBC>10 × 109/1 with blasts and rapidly progressing leukocytosis and/or rapidly progressing extramedullary lesions

ECOG Eastern Cooperative Oncology Group performance status, AlloSCT allogeneic stem cell transplantation 
Fig. 3 Overall survival of patients achieving different responses to glasdegib + LDAraC, $p<0.001$. OS, overall survival; $\mathrm{CI}$, confidence interval; $\mathrm{CR}$, complete remission; $\mathrm{CRp}$, complete remission with incomplete platelet recovery; PR, partial remission; $\mathrm{PD}$, progressive disease; $\mathrm{SD}$, stable disease

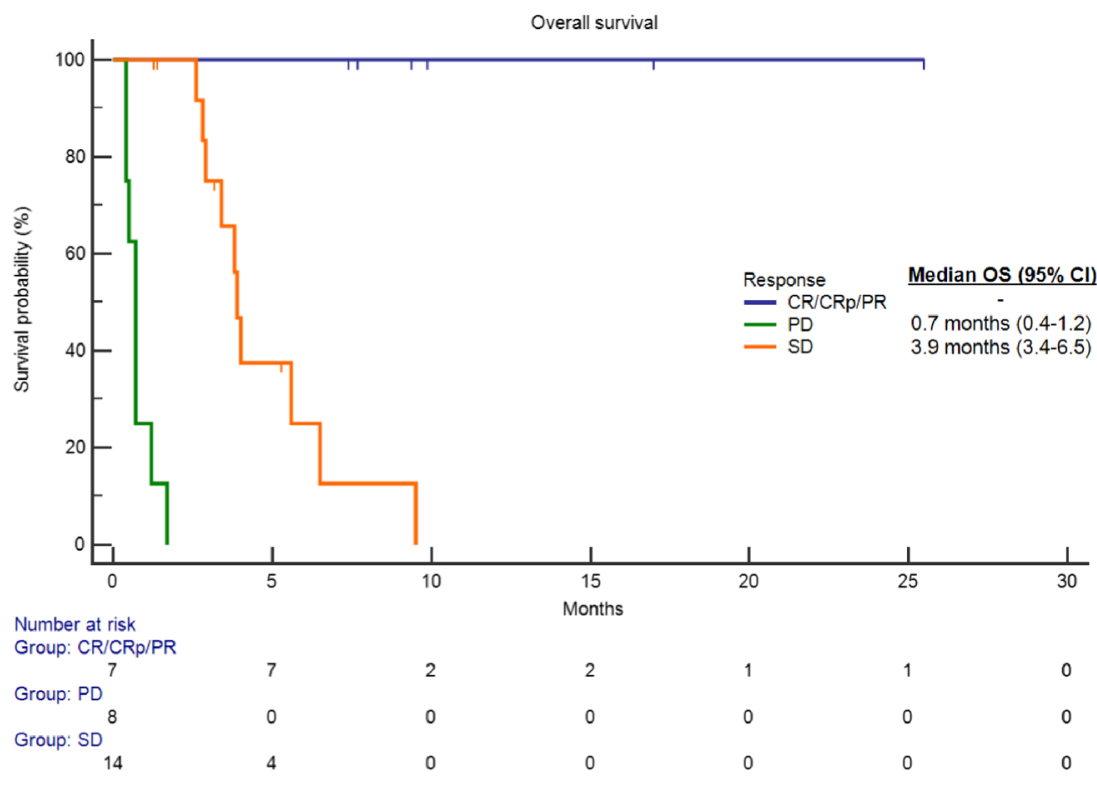

Both infectious and hemorrghagic complications during glasdegib + LDAraC treatment were mainly attributable to the uncontrolled AML, and only a minority of adverse events were treatment-related. Interestingly, QT prolongation was rare, regardless of the fact that the majority of patients received multiple concomitant QTprolonging agents (azoles, fluoroquinolones, amiodarone). Only $6 \%(2 / 31)$ of patients had grade 3 QT prolongation, and no episodes of life-threatening arrhythmias were observed. This may be partially explained by the prophylactic potassium and magnesium supplementation in the majority of cases and routine ECG evaluation. Other glasdegib-related toxicities (alopecia, dysgeusia, anorhexia, gastrointestinal symptoms, diarrhea) were also rare. Overall, glasdegib + LDAraC was tolerated well in patients achieving response or disease stabilization and was successfully administered in the outpatient setting.

Our study has limitations. This was a retrospective, nonrandomized, single-center study with a limited number of patients and a limited observation time. Nevertheless, to the best of our knowledge, this is the first report showing that glasdegib + LDAraC induces remission in poor-risk $\mathrm{R} / \mathrm{R}$ AML patients with the majority achieving at least disease stabilization.

In summary, our data demonstrate that glasdegib + LDAraC is a fairly safe, non-intensive outpatient regimen that may induce complete remission and result in prolonged survival in some R/R AML patients. Older patients after low-intensity therapy failure seem to benefit the most. Larger prospective studies are warranted.
Fig. 4 Effect of different variables on overall survival. Proliferative disease criteria: WBC $>10 \times 109 / 1$ with blasts and rapidly progressing leukocytosis and/or rapidly progressing extramedullary lesions. Lowintensity therapy:

hypomethylating agents/low-dose Cytarabine \pm venetoclax. ECOG, Eastern Cooperative Oncology Group performance status; AlloSCT, allogeneic stem cell transplantation
SUBGROUP

Age $\geq 70$

Low intensity therapy only

Adverse Cytogenetics

ECOG 2-3

Previous alloSCT

Proliferative disease

$>1$ previous treatment lines

Previous Venetoclax exposure

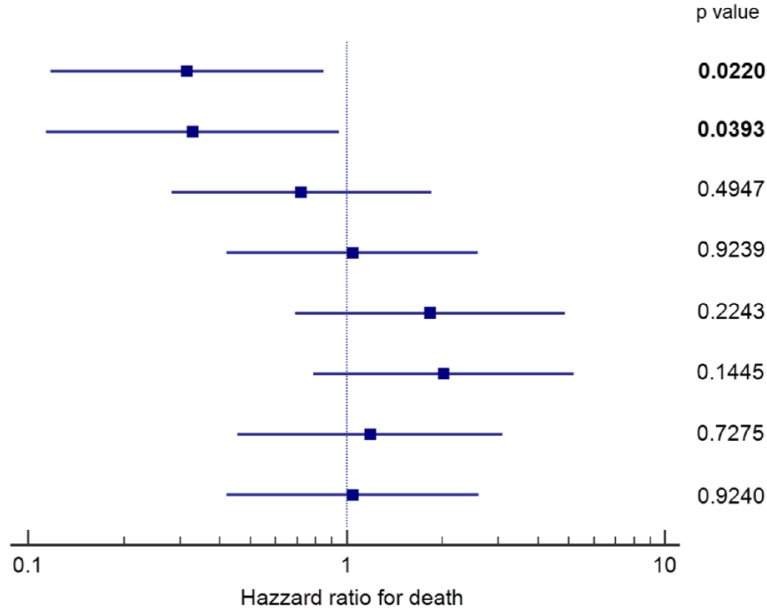


Table 4 Toxicity profile

\begin{tabular}{|c|c|c|c|c|c|c|}
\hline Adverse event & Total & Grade 1 & Grade 2 & Grade 3 & Grade 4 & Grade 5 \\
\hline \multicolumn{7}{|c|}{ Non hematological CTCAE v5.0 Grade 3-5 adverse events } \\
\hline Sepsis & $7 / 31(23 \%)$ & & & & $1 / 31(3 \%)$ & $6 / 31(19 \%)$ \\
\hline Pneumonia & $9 / 31(29 \%)$ & & & $7 / 31(23 \%)$ & $2 / 31(6 \%)$ & \\
\hline Febrile neutropenia & $5 / 31(16 \%)$ & & & $5 / 31(16 \%)$ & & \\
\hline Otitis & $1 / 31(3 \%)$ & & & & $1 / 31(3 \%)$ & \\
\hline Cl. Difficile enterocolitis & $3 / 31(10 \%)$ & & & $3 / 31(10 \%)$ & & \\
\hline Sinusitis & $1 / 31(3 \%)$ & & & $1 / 31(3 \%)$ & & \\
\hline Odontogenic infection & $3 / 31(10 \%)$ & & & $3 / 31(10 \%)$ & & \\
\hline Mucositis & $4 / 31(13 \%)$ & & & $4 / 31(13 \%)$ & & \\
\hline Skin infection & $2 / 31(6 \%)$ & & & & & \\
\hline COVID-19 infection & $2 / 31(6 \%)$ & & & $1 / 31(3 \%)$ & & $1 / 31(3 \%)$ \\
\hline Acute kidney failure & $1 / 31(3 \%)$ & & & & $1 / 31(3 \%)$ & \\
\hline Arthralgia/myalgia & $3 / 31(10 \%)$ & & & $3 / 31(10 \%)$ & & \\
\hline Fatigue & 1/31 (3\%) & & & $1 / 31(3 \%)$ & & \\
\hline Delirium & $1 / 31(3 \%)$ & & & $1 / 31(3 \%)$ & & \\
\hline Seizures & 1/31 (3\%) & & & $1 / 31(3 \%)$ & & \\
\hline Syncope & $2 / 31(6 \%)$ & & & $2 / 31(6 \%)$ & & \\
\hline Capillary leak syndrome & $1 / 31(3 \%)$ & & & $1 / 31(3 \%)$ & & \\
\hline Ascites & $1 / 31(3 \%)$ & & & $1 / 31(3 \%)$ & & \\
\hline Hypokalemia & 1/31 (3\%) & & & $1 / 31(3 \%)$ & & \\
\hline Gastrointestinal bleeding & $2 / 31(6 \%)$ & & & $2 / 31(6 \%)$ & & \\
\hline Epistaxis & $4 / 31(10 \%)$ & & & $4 / 31(13 \%)$ & & \\
\hline CNS bleeding & 1/31 (3\%) & & & & & $1 / 31(3 \%)$ \\
\hline Intraabdominal bleeding & $1 / 31(3 \%)$ & & & & & $1 / 31(3 \%)$ \\
\hline Oral hemorrhage & $2 / 31(6 \%)$ & & & $2 / 31(6 \%)$ & & \\
\hline Other hemorrhages & $2 / 31(6 \%)$ & & & $2 / 31(6 \%)$ & & \\
\hline \multicolumn{7}{|c|}{ Adverse events of special interest } \\
\hline QT prolongation & $4 / 31(13 \%)$ & $2 / 31(6 \%)$ & & $2 / 31(6 \%)$ & & \\
\hline Alopecia & $3 / 31(10 \%)$ & $3 / 31(10 \%)$ & & & & \\
\hline Dysgeusia & $1 / 31(3 \%)$ & $1 / 31(3 \%)$ & & & & \\
\hline Anorexia & $2 / 31(6 \%)$ & & & 2/31 (6\%) & & \\
\hline Gastritis & 1/31 (3\%) & & & $1 / 31(3 \%)$ & & \\
\hline Duodenal ulcer & $2 / 31(6 \%)$ & & & $2 / 31(6 \%)$ & & \\
\hline Diarrhea & $2 / 31(6 \%)$ & & $2 / 31(6 \%)$ & & & \\
\hline Gastric ulcer & 1/31 (3\%) & & & & $1 / 31(3 \%)$ & \\
\hline Esophagitis & 1/31 (3\%) & & $1 / 31(3 \%)$ & & & \\
\hline
\end{tabular}

$C N S$, central nervous system; CTCAE, Common Terminology Criteria for Adverse Events

The grey shading refers to "Not evaluated" 
Code availability (Software application or custom code): not applicable.

Author contribution All authors had contributed to the study.

Data availability Availability of data and material: the data that support the findings of this study are available on request from the corresponding author.

\section{Declarations}

Ethics approval and consent to participate The study has been approved by the Vilnius Regional Biomedical Research Ethics Committee and has therefore been performed in accordance with the ethical standards laid down in the 1964 Declaration of Helsinki and its later amendments. All procedures followed were in accordance with the ethical standards of the Vilnius Regional Biomedical Research Ethics Committee on human experimentation (institutional and national) and with the Helsinki Declaration of 1975, as revised in 2008. Informed consent was obtained from all patients for being included in the study.

Consent for publication All authors consent to the publication.

Competing interests The authors declare no competing interests.

\section{References}

1. Ferrara F, Lessi F, Vitagliano O, Birkenghi E, Rossi G (2019) Current therapeutic results and treatment options for older patients with relapsed acute myeloid leukemia. Cancers (Basel) 11(2):224. https://doi.org/10.3390/cancers 11020224

2. Bryan JC, Jabbour EJ (2015) Management of relapsed/refractory acute myeloid leukemia in the elderly: current strategies and developments. Drugs Aging 32(8):623-637

3. Daver N, Wei AH, Pollyea DA, Fathi AT, Vyas P, DiNardo CD (2020) New directions for emerging therapies in acute myeloid leukemia: the next chapter. Blood Cancer J 10(10):1-12

4. DiNardo CD, Perl AE (2019) Advances in patient care through increasingly individualized therapy. Nat Rev Clin Oncol 16(2): 73-74

5. Thomas X, Heiblig M (2020) An evaluation of glasdegib for the treatment of acute myelogenous leukemia. Expert Opin Pharmacother 21(5):523-530

6. Rimkus TK, Carpenter RL, Qasem S, Chan M, Lo HW (2016) Targeting the sonic hedgehog signaling pathway: review of smoothened and GLI inhibitors. Cancers 8(2):22. https://doi.org/ 10.3390 /cancers 8020022

7. Terao T, Minami Y (2019) Targeting hedgehog (Hh) pathway for the acute myeloid leukemia treatment. Cells 8(4):312. https://doi. org $/ 10.3390 /$ cells 8040312

8. Norsworthy KJ, By K, Subramaniam S, Zhuang L, Valle PLD, Przepiorka D et al (2019) FDA approval summary: glasdegib for newly diagnosed acute myeloid leukemia. Clin Cancer Res 25(20): 6021-6025

9. Cortes JE, Heidel FH, Hellmann A, Fiedler W, Smith BD, Robak T, Montesinos P, Pollyea DA, DesJardins P, Ottmann O, Ma WW, Shaik MN, Laird AD, Zeremski M, O'Connell A, Chan G, Heuser M (2019) Randomized comparison of low dose cytarabine with or without glasdegib in patients with newly diagnosed acute myeloid leukemia or high-risk myelodysplastic syndrome. Leukemia. 33(2): 379-389

10. Cortes JE, Douglas Smith B, Wang ES, Merchant A, Oehler VG, Arellano M, DeAngelo DJ, Pollyea DA, Sekeres MA, Robak T, Ma WW, Zeremski M, Naveed Shaik M, Douglas Laird A, O'Connell A, Chan G, Schroeder MA (2018) Glasdegib in combination with cytarabine and daunorubicin in patients with AML or high-risk MDS: phase 2 study results. Am J Hematol 93(11):1301-1310

11. Cortes JE, Dombret H, Merchant A, Tauchi T, DiRienzo CG, Sleight B et al (2019) Glasdegib plus intensive/nonintensive chemotherapy in untreated acute myeloid leukemia: BRIGHT AML 1019 Phase III trials. Future Oncol 15(31):3531-3545

12. Döhner H, Estey E, Grimwade D, Amadori S, Appelbaum FR, Büchner T et al (2017) Diagnosis and management of AML in adults: 2017 ELN recommendations from an international expert panel. Blood 129(4):424-447

13. Tavares M, Chacim S, Mariz JM (2020) Compassionate use of glasdegib in combination with low-dose cytarabine for relapsed, refractory acute myeloid leukemia or high-risk myelodysplastic syndrome. Ann Hematol [cited 2021 Jan 11]; Available from: https://doi.org/10.1007/s00277-020-04291-0

14. Sallman DA, Komrokji RS, Sweet KL, Mo Q, McGraw KL, Duong $\mathrm{VH}$ et al (2019) A phase 2 trial of the oral smoothened inhibitor glasdegib in refractory myelodysplastic syndromes (MDS). Leuk Res 81:56-61

15. Cortes JE, Heidel FH, Fiedler W, Smith BD, Robak T, Montesinos P, Candoni A, Leber B, Sekeres MA, Pollyea DA, Ferdinand R, Ma WW, O'Brien T, O'Connell A, Chan G, Heuser M (2020) Survival outcomes and clinical benefit in patients with acute myeloid leukemia treated with glasdegib and low-dose cytarabine according to response to therapy. J Hematol Oncol 13(1):92

16. Kubasch AS, Platzbecker U (2019) The wolf of hypomethylating agent failure: what comes next? Haematologica. 104(8):1505-1508

17. Prébet T, Gore SD, Esterni B, Gardin C, Itzykson R, Thepot S, Dreyfus F, Rauzy OB, Recher C, Adès L, Quesnel B, Beach CL, Fenaux P, Vey N (2011) Outcome of high-risk myelodysplastic syndrome after azacitidine treatment failure. J Clin Oncol 29(24): 3322-3327

18. Komrokji RS (2015) Treatment of higher-risk myelodysplastic syndromes after failure of hypomethylating agents. Clin Lymphoma Myeloma Leuk 15:S56-S59

19. Zucenka A, Pileckyte R, Trociukas I, Peceliunas V, Vaitekenaite V, Maneikis K, et al. Outcomes of relapsed or refractory acute myeloid leukemia patients failing venetoclax-based salvage therapies. Eur J Haematol [cited 2020 Nov 2];n/a(n/a). Available from: https://doi. org/10.1111/ejh.13527

Publisher's Note Springer Nature remains neutral with regard to jurisdictional claims in published maps and institutional affiliations. 